\title{
Human HTm4 is a hematopoietic cell cycle regulator
}

\author{
José L. Donato, ${ }^{1}$ Jon Ko, ${ }^{1}$ Jeffery L. Kutok, ${ }^{2}$ Tao Cheng, ${ }^{3}$ Taro Shirakawa, ${ }^{4,5,6}$ \\ Xiao-Quan Mao, ${ }^{5}$ David Beach, ${ }^{7}$ David T. Scadden, ${ }^{8}$ Mohamed H. Sayegh, ${ }^{9,10}$ \\ and Chaker N. Adra ${ }^{1,10,11}$
}

${ }^{1}$ Departments of Medicine, Division of Hematology and Oncology, Beth Israel Deaconess Medical Center,
Harvard Medical School, Boston, Massachusetts, USA
${ }^{2}$ Department of Pathology, Brigham and Women's Hospital, Harvard Medical School, Boston, Massachusetts, USA
${ }^{3}$ Department of Radiation Oncology, and University of Pittsburgh Cancer Institute, University of Pittsburgh,
Pittsburgh, Pennsylvania, USA
${ }^{4}$ Experimental Medicine Unit, University of Wales Swansea, Swansea, Wales, United Kingdom
${ }^{5}$ Department of Health Promotion and Human Behavior, Kyoto University Graduate School of Public Health, Kyoto, Japan
${ }^{6}$ RIKEN SNP Typing Center, Tokyo, Japan
${ }^{7}$ Genetica Inc., Cambridge, Massachusetts, USA
${ }^{8}$ Experimental Hematology, AIDS Research Center, Massachusetts General Hospital, Boston, Massachusetts, USA
${ }^{9}$ Laboratory of Immunogenetics and Transplantation, Brigham and Women's Hospital, Harvard Medical School,
Boston, Massachusetts, USA
${ }^{10}$ Division of Nephrology, Children's Hospital, Harvard Medical School, Boston, Massachusetts, USA
${ }^{11}$ Department of Pathology, Beth Israel Deaconess Medical Center, Harvard Medical School, Boston, Massachusetts, USA

Address correspondence to: C. Adra, Department of Medicine, Beth Israel Deaconess Medical Center, 99 Brookline Avenue, Boston, Massachusetts, 02215. Phone: (617) 667-3766; Fax: (617) 975-7905; E-mail: cadra@caregroup.harvard.edu.

Jose L. Donato and Jon Ko contributed equally to this work.

Received for publication August 22, 2001, and accepted in revised form November 13, 2001.

Proper control of cell cycle progression is critical for the constant self-renewal, differentiation, and homeostasis of the hematopoietic system. Cells of all types share the common cell cycle regulators. The different expression patterns of common regulators, in a broad sense, define cell-type or lineage specificity. However, there remains the possibility of hematopoietic cell cycle regulators tailored to the demands of the hematopoietic system. Here we describe a novel protein, HTm4, which serves as a hematopoietic cell cycle regulator. Our data indicate that HTm 4 is expressed in hematopoietic tissues and is tightly regulated during the differentiation of hematopoietic stem cells. It binds to cyclin-dependent kinase-associated (CDK-associated) phosphatase-CDK2 (KAP-CDK2) complexes, and the three proteins demonstrate similar patterns of cellular expression in human lymphoid tissues. HTm 4 stimulates the phosphatase activity of KAP, and its C-terminal region is required for binding to KAP-CDK2 complexes and the modulation of KAP activity. Overexpression of $\mathrm{HTm} 4$ can cause cell cycle arrest at the $\mathrm{G}_{0} / \mathrm{G}_{1}$ phase. Thus, $\mathrm{HTm} 4$ is a novel hematopoietic modulator for the $\mathrm{G}_{1}-\mathrm{S}$ cell cycle transition.

J. Clin. Invest. 109:51-58 (2002). DOI:10.1172/JCI200214025.

\section{Introduction}

Cell cycle progression and hematopoiesis are tightly coordinated $(1,2)$. The maintenance of proper hematopoiesis requires a balance between the proproliferative and antiproliferative properties of hematopoietic stem cells (HSCs). This balance can be biologically translated into the known capabilities of HSCs to selfrenew, differentiate, and generate the entire population of hematopoietic cells of all lineages. During cell cycle progression, several antiproliferative signals are activated (3-6). Antiproliferative properties of HSCs serve important biological functions that, in addition to the preservation of integrity of genetic materials, include the preservation of multipotential, early progenitor cells $(7,8)$, their differentiation to the committed late progenitor cells $(9,10)$, and subsequent maturation to terminally differentiated hematopoietic cells.

The temporal expression of various cyclins and cyclindependent kinases (CDKs) is the foundation upon which many cell cycle modulators exert their influence (3-6). The inhibition of the kinase activities of CDK2, 4, and 6 is known to cause $G_{0} / G_{1}$ cell cycle arrest (reviewed in ref. 11), which is believed to be important for the differentiation and maturation of hematopoietic cells. This inhibition is achieved primarily through the binding of inhibitors to CDKs. The cyclin D-CDK4/6 complexes play an important role in the progression or restriction of cell cycle progression at the $G_{1}$ phase. Inhibition of

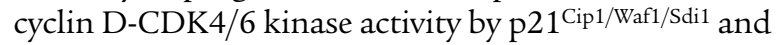
p27 ${ }^{\text {Kip } 1}$ inhibitors at this restriction point causes $G_{0} / G_{1}$ cell cycle arrest. Cyclin E-CDK2 and cyclin A-CDK2 are responsible for the $\mathrm{G}_{1}-\mathrm{S}$ phase transition (12) and $S$ phase progression of the cell cycle, respectively. Both complexes are susceptible to negative regulation by $\mathrm{p} 21^{\mathrm{Cip} 1 / \text { Waf1/Sdi1 }}$ and $\mathrm{p} 27^{\mathrm{Kip} 1}$. Interference with cyclin E-CDK2 activity can cause $G_{0} / G_{1}$ cell cycle arrest. With the involvement of cyclin D-CDK4/6, the interplay between $\mathrm{p}^{27^{\mathrm{Kip}} 1}$ and cyclin E-CDK2 is complex, varying according to the dif- 
ferent physiological conditions of the cell (reviewed in ref. 3 ). In a rapidly cycling cell, most of $\mathrm{p} 27^{\mathrm{Kip} 1}$ is degraded, and the residual amount of $\mathrm{p} 27^{\mathrm{Kip} 1}$ is sequestered by cyclin $\mathrm{D}-\mathrm{CDK} 4 / 6$, thus alleviating its negative regulation upon cyclin E-CDK2. However, in the absence of mitogen, the concentration of $\mathrm{p} 27^{\mathrm{Kip} 1}$ protein increases while that of cyclin $\mathrm{D}$ decreases. This combined effect can result in rapid inactivation of cyclin E-CDK2 kinase and bring about $\mathrm{G}_{0} / \mathrm{G}_{1}$ cell cycle arrest in just one cell cycle. Due to their ability to modulate the kinase activities of CDKs,

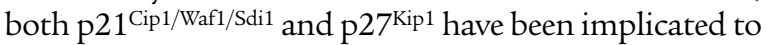
be involved in many aspects of hematopoiesis (7-10), from stem cell kinetics to terminal differentiation and maturation of hematopoietic cells.

The monomeric, phosphorylated CDK2 has only a basal level of kinase activity. Activation of CDK2 kinase activity requires both the phosphorylation of threonine residue $\left(\mathrm{Thr}^{160}\right.$ ) by CDK-activating kinase (CAK) and its binding to the cyclins (13-15). CAK can phosphorylate both CDK2 monomer and cyclins-CDK2 complex. Dephosphorylation of the active phosphorylthreonine residue of CDK2 inactivates its intrinsic kinase activity, representing yet another control mechanism for cell cycle progression. KAP, a CDK-associated phosphatase, can dephosphorylate $\mathrm{Thr}^{160}$ in human CDK2 (16). It binds to CDK2 both in the presence and absence of cyclins (16-18), but can only dephosphorylate CDK2 in the absence of cyclins. The binding of KAP to the cyclinsCDK2 complex does not interfere with the kinase activity. It seems the function of KAP is to counter CAK in two ways: (a) dephosphorylation of monomeric CDK2 after it is phosphorylated by CAK and (b) interference with CAK kinase activity by binding to CDK2 or the cyclins-CDK2 complex. The biological significance of $\mathrm{KAP}$ is not as well established as that of CDK inhibitors. Nevertheless, it has been shown that exogenous expression of KAP slows the $\mathrm{G}_{1}$ phase cell cycle progression in HeLa cells (17) and that aberrant KAP transcripts are detected in some hepatocellular carcinomas (19). These observations suggest that KAP has the same biological effects as that of CDK inhibitors, although their modes of action are different.

Although the expression patterns of some common cell cycle regulators vary among hematopoietic cells of different lineages $(10,20)$, all the components of cell cycle regulation thus far discussed are common to all cell types. Here we report a novel protein, HTm4, which acts as a hematopoietic cell cycle regulator. $\mathrm{HTm} 4$ is a member of HTm $4 / C D 20 / F c E R I \beta$ subfamily with four transmembrane domains (21). It is expressed in hematopoietic cells, including bone marrow cells. It has been found to be associated with atopic asthma (22). HTm4, KAP, and CDK2 form a physiologic complex, the in vivo presence of which is confirmed by their similar patterns of cellular expression in human lymphoid tissue. Our data demonstrate that HTm 4 modulates the level of phosphorylation of CDK2 through its direct binding to KAP. The exogenous expression of HTm 4 in U937 cells causes $\mathrm{G}_{0} / \mathrm{G}_{1}$ cell cycle arrest.
Thus, the cell cycle regulatory pathway involving the dephosphorylation of CDK2 has been expanded to include $\mathrm{HTm} 4$, and the participation of KAP in hematopoiesis is implicated.

\section{Methods}

In vitro cell culture and transfection. The isolation of bone marrow cells and the subsequent manipulations of the isolated stem cells were carried out as described previously (23). Cells at various stages of differentiation were isolated as single cells and then subjected to RT-PCR analysis for the expression of HTm4. The induction of differentiation of $\mathrm{CD} 34^{+}$stem cells was also done in the presence of $300 \mathrm{U} / \mathrm{ml}$ of granulocyte colony stimulating factor (G-CSF) or $3 \mathrm{U} / \mathrm{ml}$ of erythropoietin (Epo) for the period of time as specified (24). Transient expression of hemagglutinin-KAP (HA-KAP) was done as follows: $5 \mu \mathrm{g}$ per $10^{7}$ cells of PCMV-HA-KAP (18) and the control PCMV vectors were transfected into U937 and KU812, using Superfect reagent (QIAGEN, Valencia, California, USA). Twenty-four hours after transfection, cell lysates were prepared from both samples. U937 and KU812 were routinely maintained in RPMI-1640 medium supplemented with $10 \%$ (vol/vol) FBS, 2 mM glutamine, 100 $\mathrm{IU} / \mathrm{ml}$ penicillin, and $100 \mu \mathrm{g} / \mathrm{ml}$ streptomycin in an atmosphere of $5 \% \mathrm{CO}_{2}$, at $37^{\circ} \mathrm{C}$.

Cell fractionation and preparation of cell lysates. Fractionation of the subcellular components of U937 or KU812 was done as follows: cells were resuspended in ice-cold PBS with Protease Cocktail Tablets (Roche Molecular Biochemicals, Mannheim, Germany) and homogenized in a Douce glass homogenizer. The extract was centrifuged at $200 \mathrm{~g}$ for 10 minutes at $4^{\circ} \mathrm{C}$ to pellet the nuclei. The supernatant was clarified further by centrifugation at $15,000 \mathrm{~g}$ for 30 minutes at $4^{\circ} \mathrm{C}$. The pellet was considered to be the membrane fraction. Total lysate, nuclear, and membrane fractions were used for the Western blot analysis. For the production of U937 and KU812 cell lysates, we extracted cells $\left(10^{6} \mathrm{cells} / \mathrm{ml}\right)$ in PBS containing $0.5 \%$ Triton X-100 and Protease Cocktail Tablets at $4^{\circ} \mathrm{C}$ for 30 minutes. The clear lysates were collected after centrifugation at $4^{\circ} \mathrm{C}$, $10,000 \mathrm{~g}$ for 30 minutes and kept on ice before use.

Expression analysis in single cells using RT-PCR. Individual cells were obtained by means of a Becton Dickinson Immunocytometry Systems (San Jose, California, USA) FACS cell sorter, by limiting dilution analysis as described by Taswell (25). cDNAs representative of the total mRNA isolated from individual cells were synthesized by a micro-reverse transcription reaction using oligo-dT primers as described by Berardi et al. (23). The PCR products were analyzed by Southern blot technique (26) using $\alpha^{32} \mathrm{P}$-dCTP-labeled HTm 4 cDNA probe.

Yeast two-bybrid system. Bait consisting of the last 25 amino acids of $\mathrm{HTm} 4\left(\mathrm{C}_{\mathrm{HTm}} 4\right)$, from amino acid 190 to 214, was cloned into the vector PVJLII (27). The yeast strain L40 containing two reporter genes, HIS3 and LacZ, was used as the host for a two-hybrid assay. Plas- 
mid vector was cotransformed with the human bone marrow cDNA-pGADGH library (CLONTECH, San Diego, California, USA) into L40. Transformants were plated onto synthetic medium lacking histidine, leucine, and tryptophan. After 10 days of growth, $\mathrm{His}^{+}$ colonies were patched onto selection plates and tested for $\beta$-galactosidase activity. Plasmid DNAs were isolated from positive clones and introduced by electroporation into Escherichia coli MH4 plated on leucine-free medium for the selection of the PGADGH construct. The putative positive cDNAs in $\mathrm{pGADGH}$ plasmid were then tested in a two-hybrid assay with irrelevant, negative controls. The negative controls used were $\operatorname{MEK}(28)$, byr (29), and lamin (27). The identified cDNA clones in pGADGH constructs were considered true positive when they were tested negative with the negative controls. To make sure that the interactions between HTm 4 and its target proteins were not permutation dependent, the entire two-hybrid assay was repeated with constructs that had their expression vectors exchanged between two groups. In this case, HTm 4 was subcloned into PGADGH while KAP into the vector PVJLII.

Generation of anti-HTm 4 polyclonal Ab. A peptide, GSLQYPYHFQKHF, from the HTm4 sequence between the first and the second predicted transmembrane domains (amino acids 70 to 82), was synthesized with the amino-terminal cysteine added to facilitate coupling and purification procedures. The antigen was prepared and injected into 3- to 9-month-old rabbits to raise antiserum following established protocols (Zymed Laboratories Inc., South San Francisco, California, USA). The specific Ab's were purified using an epoxy-activated Sepharose 6B coupled with the peptide as described by the manufacturer (Amersham Pharmacia Biotech Europe GmbH, Uppsala, Sweden). Please note that our HTm $4 \mathrm{Ab}$ is only suitable for Western blot analysis and cell staining. Thus, for the immunoprecipitation of both the full-length and truncated HTm 4 that are exogenously expressed in Flag-tagged forms, we used anti-Flag from Sigma Chemical Co. (St. Louis, Missouri, USA).

Flow cytometry analysis. To determine the cellular expression (cell surface versus intracellular localization) of HTm4, intact U937 $\left(10^{7}\right.$ cells $\left./ \mathrm{ml}\right)$ was labeled with $2 \mu \mathrm{g} / \mathrm{ml}$ of polyclonal anti-HTm 4 for 20 minutes, followed by the incubation with goat anti-rabbit IgGFITC-conjugated $\mathrm{Ab}$. For the staining of intracellular proteins, cells were permeabilized with the Fix \& Cell Permeabilization Kit, as described by the manufacturer (Caltag Laboratories Inc., Burlingame, California, USA). The flow cytometry analysis was performed on a FACScan flow cytometer (Becton Dickinson Immunocytometry Systems). For the absorption with the peptide (amino acids 70-82) described above, $2 \mu \mathrm{g} / \mathrm{ml}$ of $\mathrm{HTm} 4 \mathrm{Ab}$ was treated with $10 \mu \mathrm{g}$ of peptide at room temperature with continuous rotation for 30 minutes before the staining of cells.

Immunohistochemistry and immunoprecipitation. The cell preparation for immunohistochemistry study was per- formed as described (30) with some modifications. Briefly, U937 cells were allowed to adhere to polylysine precoated coverslips for 30 minutes, fixed with $2 \%$ formaldehyde in PBS, and permeabilized in $0.1 \%$ saponin and $1 \times$ PBS. All subsequent washes and Ab hybridization were carried out in the presence of saponin-PBS buffer. Cells were prehybridized with $2 \%$ BSA, incubated with $0.2 \mu \mathrm{g} / \mathrm{ml}$ anti-KAP followed by $1 \mu \mathrm{g} / \mathrm{ml}$ anti-HTm 4 for 20 minutes at room temperature. The positive staining was visualized with goat anti-mouse IgG Texas Red-conjugated or goat antirabbit IgG FITC-conjugated secondary Ab (Jackson ImmunoResearch Laboratories Inc., West Grove, Pennsylvania, USA). For immunoprecipitation, cell lysates equivalent to $5 \times 10^{5}$ cells in $0.5 \mathrm{ml}$ of lysis buffer, $5 \mu \mathrm{g}$ of $A b$, and $40 \mu l$ of protein G Sepharose beads (Sigma Chemical Co.) were used per reaction.

Western blot analysis. Enhanced chemiluminescence detection reagents were purchased from Amersham Pharmacia Biotech (Piscataway, New Jersey, USA). Primary mAb's used were anti-KAP, anti-CDK2, both from BD Transduction Laboratories ( San Diego, California, USA), anti-HA from Covance Research Products Inc. (Richmond, California, USA), and anti-Flag from Sigma Chemical Co. Secondary Ab's, horseradish peroxidase-conjugated (HRP-conjugated) goat anti-rabbit and rabbit anti-mouse Ab's, were from Amersham Pharmacia Biotech (Piscataway, New Jersey, USA). All primary Ab's were used at $1 \mu \mathrm{g} / \mathrm{ml}$ and the secondary Ab's at 1:5,000 dilution.

Isolation of KAP using GST-C $\mathrm{C}_{H T m}$ fusion protein. $\mathrm{PGEX}-4 \mathrm{~T}$ $\mathrm{C}_{\mathrm{HTm} 4}$ vector was used for the production of GST- $\mathrm{C}_{\mathrm{HTm} 4}$. This expression vector contained the C-terminal region of HTm 4 that coded for the amino acid 190 to 214 $\left(\mathrm{C}_{\mathrm{HTm}} 4\right)$, with in-frame reading for GST protein at the $5^{\prime}$ end, using pGEX-4T-1 vector (Amersham Pharmacia Biotech, Piscataway, New Jersey, USA). The production and purification of GST- $\mathrm{C}_{\mathrm{HTm}} 4$ was done according to the protocols specified by the manufacturer (Amersham Pharmacia Biotech, Piscataway, New Jersey, USA). GST protein without the fusion partner was used as control. One milliliter each of U937 and KU812 lysates was incubated with $5 \mu \mathrm{g}$ of GST- $\mathrm{C}_{\mathrm{HTm} 4}$ or GST coupled to glutathione Sepharose beads for 4 hours at $4^{\circ} \mathrm{C}$ and analyzed by Western blot technique.

Tet-Offexpression and induction of cell cycle arrest. The induction of HTm 4 expression was done using the Tet-Off expression system of CLONTECH for the studies of its interaction with KAP, Cdk2, and induced cell cycle arrest. HTm 4 proteins in both full-length and C-terminal truncated (without the last 22 amino acids, denoted as HTm4-C) forms were used for sideby-side comparison studies. The maintenance and induction of cell culture were carried out according to manufacturer's protocols. In brief, to generate cell clones expressing the inducible HTm 4 constructs we constructed HTm 4 and its variants in the PTRE2 vector with an in-frame C-terminal Flag epitope tag. U937 cells expressing the tetracycline-controlled transacti- 
a

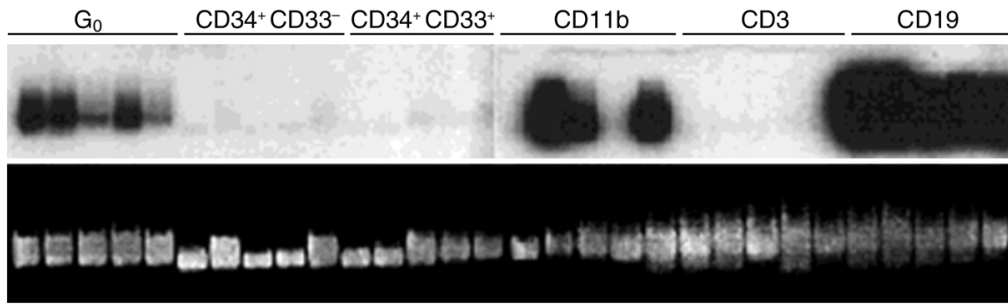

b

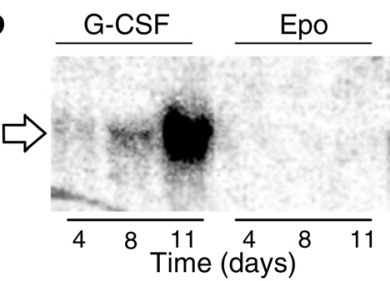

Figure 1

The expression of HTm4 is highly regulated during the differentiation of hematopoietic stem cells. (a) MicroRT-PCR analysis of HTm4 expression in single hematopoietic cells at various stages of differentiation. Cell surface markers for each sample are denoted on the top row. $\mathrm{G}_{0}$ represents the quiescent CD34 ${ }^{+}$CD38- HSC. The middle row is a Southern blot analysis of HTm 4 cDNA obtained through RT-PCR technique. The bottom row shows the loaded cDNA stained with ethidium bromide dye. Each lane represents a single cell. (b) Northern blot analysis of HTm4 expression in $\mathrm{CD} 34^{+}$cells that were induced to differentiate with the treatment of either G-CSF or Epo for time intervals as indicated. At day 11, cell populations are determined to be $60 \%$ neutrophils and 70\% erythrocytes when induced with G-CSF and Epo, respectively.

vator were used as the host. Cells were cotransfected by electroporation with $5 \mu \mathrm{g}$ of expression vector and $0.25 \mu \mathrm{g}$ of $\mathrm{pHyg}$ plasmid containing the hygromycine resistance gene per $10^{7}$ cells. Positive clones were maintained in medium containing $200 \mu \mathrm{g} / \mathrm{ml}$ hygromycin and $1 \mu \mathrm{g} / \mathrm{ml}$ of doxycycline (Dox). In the Tet-Off system, the expression of HTm 4 and HTm4-C proteins were induced in the absence of Dox, while their suppressions were achieved in the presence of Dox $(1 \mu \mathrm{g} / \mathrm{ml})$. Cells were synchronized as described by Ling et al. (31). Briefly, $10^{6} / \mathrm{ml}$ of cells was treated with two rounds of incubation with $2 \mathrm{mM}$ thymidine for 16 hours each, separated by 10 hours of resting period in the absence of thymidine. The first thymidine treatment was done in the presence of $10 \%$ FBS and the second in $0.5 \%$ FBS. The stimulation of cell growth, after synchronization, was done in the presence of $15 \%$ FBS. Cell samples analyzed at the time points indicated were fixed with $70 \%$ alcohol at $4{ }^{\circ} \mathrm{C}$ for 30 minutes, stained with propidium iodide $(50 \mu \mathrm{g} / \mathrm{ml})$, and then analyzed by flow cytometry.

\section{Figure 2}

(a) Binding of $\mathrm{HTm} 4$ to KAP in yeast two-hybrid assay. Constructs listed in the upper and lower rows were in PGADGH, whereas those to the left of the first column were in PVJLII. HTm4 listed here represents the $\mathrm{C}_{\mathrm{HTm} 4}$. MEK, byr, and lamin are negative controls. The positive results are shown as colony growth on selective medium and blue color reaction in the presence of $\beta$-gal. (b) Isolation of KAP from U937 and KU812 lysates using GST- $\mathrm{C}_{\mathrm{HTm} 4}$ fusion protein. GST-C represents $\mathrm{GST}_{\mathrm{H}} \mathrm{C}_{\mathrm{HTm} 4}$. Lysates derived from U937 and KU812 are absorbed with either GST- $\mathrm{C}_{\mathrm{HTm} 4}$ or GST-coupled beads, and bound proteins were analyzed in a Western blot assay for the presence of KAP. (c) Coimmunoprecipitation of HA-tagged KAP and HTm4. CTL, pCMV control vector. The source of the samples is listed on the top. The first two columns on the left are lysates alone and the remaining two are after immunoprecipitation with anti-HA. Samples are analyzed by the Western blot technique using anti-HTm4 for the top two panels and anti-HA for the bottom two. The identified proteins are listed on the right. (d) HTm4 and KAP form a physiological complex. The source of the samples is denoted on the top; the first column from the left is U937 lysate alone; the second and third are after immunoprecipitation with anti-KAP and IgG control, respectively. The presence of $\mathrm{HTm} 4$ is indicated on the right after Western blot analysis. Representative figures of at least five experiments.

Immunohistochemical studies. All staining was performed by standard immunoperoxidase methods. Briefly, slides were deparaffinized and were either not pretreated (HTm4), pretreated in 1 mM EDTA, $\mathrm{pH} 8.0$, for 20 minutes at $95^{\circ} \mathrm{C}(\mathrm{Ki}-67)$, or pretreated in $10 \mathrm{mM}$ sodium citrate, $\mathrm{pH} 6.0$, for 20 minutes at $95^{\circ} \mathrm{C}(\mathrm{CDK} 2$ and KAP). All further steps were performed at room temperature in a hydrated chamber. Slides were pretreated with Peroxidase Block (DAKO Corp., Carpinteria, California, USA) for 5 minutes to quench endogenous peroxidase activity, and a 1:5 dilution of goat serum in $50 \mathrm{Mm}$ Tris-Cl, $\mathrm{pH}$ 7.4, for 20 minutes to block nonspecific binding sites. Either affinity-purified rabbit anti-HTm $4 \mathrm{Ab}$ (1:1,000 dilution in $50 \mathrm{mM}$ Tris$\mathrm{Cl}, \mathrm{pH} 7.4$, with $3 \%$ goat serum); murine anti-CDK2 Ab (1:500 dilution in $50 \mathrm{mM}$ Tris-Cl, $\mathrm{pH} 7.4$, with $3 \%$ goat serum; BD Biosciences, PharMingen-Transduction Laboratories, San Diego, California, USA); murine anti$\mathrm{KAP} A B$ (1:1,000 dilution in $50 \mathrm{mM}$ Tris-Cl, $\mathrm{pH}$ 7.4, with $3 \%$ goat serum; BD Biosciences, PharmingenTransduction Laboratories); or murine anti-Ki-67 Ab

a

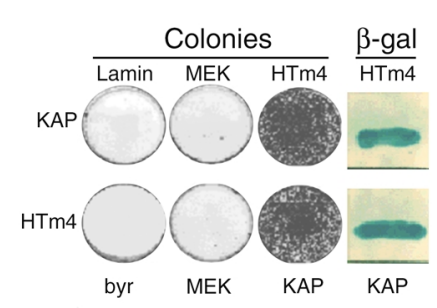

b

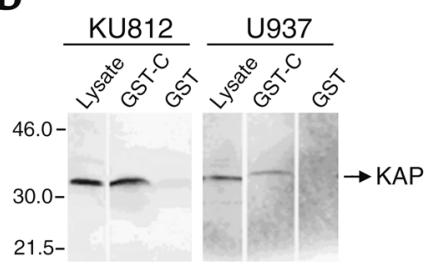

C

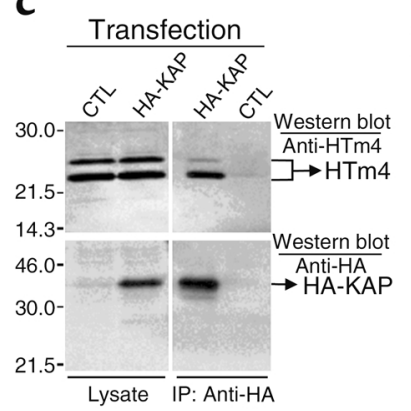

d

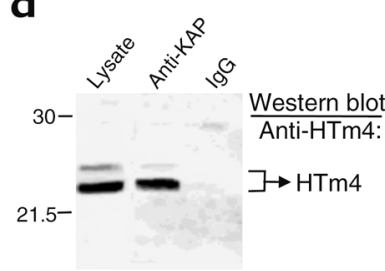


(MIB-1 clone; 1:100 dilution in $50 \mathrm{mM}$ Tris-Cl, $\mathrm{pH} 7.4$, with $3 \%$ goat serum; Coulter Corp., Miami, Florida, USA) was applied at room temperature for 1 hour. After washing in $50 \mathrm{mM}$ Tris- $\mathrm{Cl}, \mathrm{pH} 7.4$, secondary goat anti-rabbit or goat anti-mouse HRP-conjugated $\mathrm{Ab}$ (Envision detection kit; DAKO Corp.) was applied for 30 minutes. After further washing, immunoperoxidase staining was developed using a 3,3-diaminobenzidine chromogen kit (DAKO, USA) per the manufacturer and counterstaining was done with hematoxylin.

\section{Results}

Yeast two-hybrid and fusion protein assays. Using the yeast two-hybrid assay, we have detected the binding of HTm 4 to KAP (Figure 2a). The specificity of this binding has been confirmed by the isolation of KAP from U937 and KU812 lysates, using a fusion protein of GST and the last 25 amino acids of the C-terminal region of HTm4 (Figure 2b).

HTm4-KAP immunoprecipitation. The binding of HTm 4 to KAP under physiologic conditions has been established by two different approaches. First, the binding has been detected between the endogenous HTm 4 of KU812 and the exogenous HA-tagged KAP after the immunoprecipitation with anti-HA mAb (Figure 2c). Second, to eliminate the possibility that the binding between HTm4 and HA-KAP was spurious, due to the overexpression of the exogenous HA-KAP, we performed a direct immunoprecipitation of endogenous $\mathrm{KAP}$ in KU812 cells with anti-KAP Ab (Figure 2d). The resulting immune complexes have been determined to contain the endogenous $\mathrm{HTm} 4$, indicating the interaction between KAP and HTm 4 is physiologic.

HTm 4-KAP Immunohistochemistry. KAP is located predominantly in the perinuclear area (34), and in U937 HTm 4 colocalizes with KAP principally in the same region (Figure $3 \mathrm{a}$ ). This observation substantiates the physiologic interaction between HTm 4 and KAP. The subcellular location for HTm 4 has been determined to be in the microsomal membrane fraction (Figure $3 b$ ). Our anti-HTm 4 polyclonal $\mathrm{Ab}$ recognizes the native $\mathrm{HTm} 4$ as a doublet. The intracellular staining pattern (Figure $3 c$ ) is consistent across the many cells types thus far examined.

HTm4-KAP-CDK2 Immunoprecipitation. KAP is known to bind to CDK2 and $\mathrm{Cdc} 2(17,18)$. It is demonstrated here that HTm 4 binds to KAP-CDK2 complexes (Figure 4a) in immune complexes derived from U937 lysate using anti-CDK2 mAb. CDK2 is detected as doublets, with the upper band being the dephosphorylated form and the lower the phosphorylated form (Figure 4c). The immunoprecipitated complexes may be composed of solely HTm4-KAP-CDK2 or as a mixture with CDK2-KAP. In light of our previous yeast two-hybrid assay, which showed only the binding between HTm 4 and KAP, it is unlikely that HTm4 interacts directly with CDK2. The C-terminus is responsible for the binding of $\mathrm{HTm} 4$ to KAP in the yeast two-hybrid assay. Therefore, we examined whether the C-terminus of HTm4 is required for its binding to KAP-CDK2. As shown in Figure $4 \mathrm{~b}$, the $\mathrm{C}$-terminal region indeed is required for the binding of HTm 4 to KAP-CDK2 complexes.

CDK2 phosphorylation status. KAP is known to dephosphorylate Thr ${ }^{160}$ in the human CDK2 in the absence of cyclin A (16), acting as a negative regulator. It can also bind to CDK2 in the presence of cyclin A; however, the formation of cyclin A-CDK2 complexes does preclude the dephosphorylation of CDK2 by KAP. The phosphatase activity of KAP is enhanced upon the binding of HTm 4 as demonstrated in Figure 4c, which shows that the exogenous expression of full-length HTm 4 promotes the dephosphorylation of CDK2, whereas, in the presence of C-terminal truncated HTm4, the level of phosphorylated CDK2 remains unchanged. This observation implicates the modulation of CDK2 by $\mathrm{HTm} 4$ through its direct binding to KAP.

Induction of cell cycle arrest. We here evaluate the effects of $\mathrm{HTm} 4$ on cell cycle progression through the overexpression of intact HTm4 in synchronized U937 cells (Figure 5). After 20 hours of serum stimulation, 60.8\% $(n=5$; the percentage given is an average derived from five experiments) of cells, in the presence of exogenous HTm 4 , accumulate at $\mathrm{G}_{0} / \mathrm{G}_{1}$ phase of cell cycle in contrast to $35 \%(n=5)$ in the absence of exogenous HTm 4 . This effect is not detected in U937 cells with exogenous

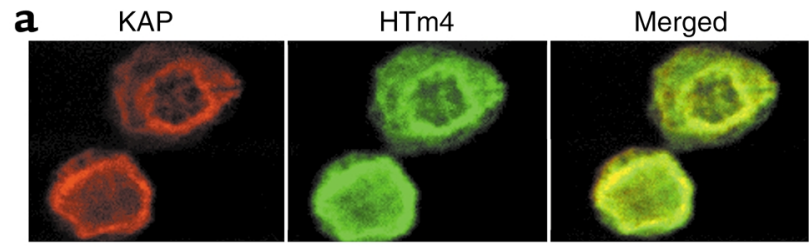

b

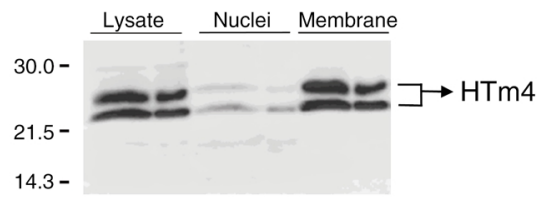

c

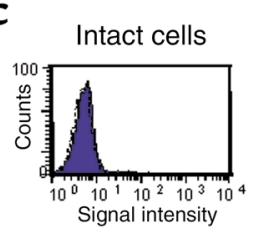

Permeabilized

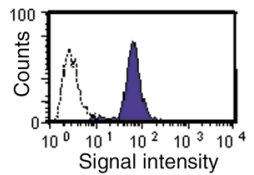

Permeabilized

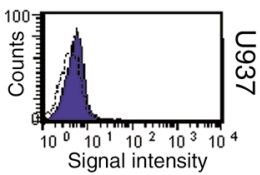

Figure 3

(a) Colocalization of HTm4 and KAP. Staining was visualized with goat anti-mouse IgG Texas Red-conjugated (left) or goat anti-rabbit IgG FITC-conjugated (middle) secondary Ab's. Merged image is given on the right showing that HTm4 and KAP colocalized predominately in the perinuclear area. (b) HTm4 is located in the intracellular membrane fraction. Source of the samples are denoted on the top row. Samples shown are in duplicates. The presence of HTm4 is indicated on the right after Western blot analysis. (c) Staining of U937 with anti$\mathrm{HTm} 4 \mathrm{Ab}$. The manipulations of samples are denoted on the top. The filled peaks represent the staining with anti-HTm4, and the open peaks represent IgG control. $x$ axis, cell counts; $y$ axis, signal intensity of FITC fluorescence. Representative figures of at least five experiments. 


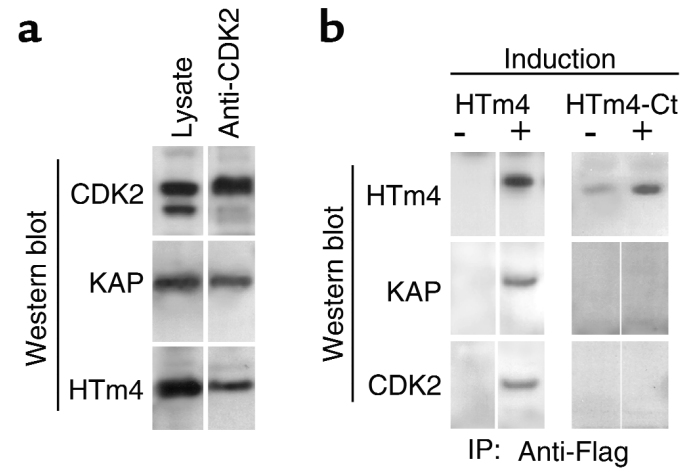

C

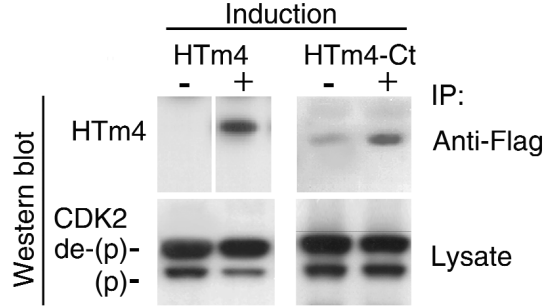

Figure 4

(a) HTm 4 binds to KAP-CDK2 under normal physiological conditions. Samples analyzed are shown on the top. The lane marked antiCDK2 includes the samples immunoprecipitated with anti-CDK2 $\mathrm{mAb}$ and analyzed by Western blot technique using Ab's as indicated on the left. (b) The $\mathrm{C}$-terminal region of $\mathrm{HTm} 4$ is required for its binding to KAP-CDK2. HTm4-Ct, HTm4 without the last 22 amino acids. Ab's used for the Western blot analysis, after immunoprecipitation with anti-Flag, are listed on the left. The induction of protein expression in the absence of Dox is marked as +, and no induction in the presence of Dox is marked as -. (c) The C-terminal region of $\mathrm{HTm} 4$ is required for the enhancement of the phosphatase activity of KAP. The descriptions for $c$ are the same as Figure $4 b$, except the top panels show the immunoprecipitation with anti-Flag $A b$ and the bottom panels are derived from $50 \mu \mathrm{g}$ of total lysate per sample. The upper bands in the lower panels are the dephosphorylated form of CDK2, marked as de- $(P)$, and the lower bands are the phosphorylated $(P)$ form. A reduction in intensity can be seen in the phosphorylated CDK2 in the presence of overexpressed Flag-HTm4. Representative figures of at least five experiments.

C-terminal truncated HTm4, 36.3\% versus 34.4\% (induced vs. noninduced; $n=5$ for both).

Immunohistochemical studies. We then studied the expression of CDK2, KAP, and HTm4 in human tissues using immunostaining with anti-CDK2, anti-KAP, and antiHTm 4 Ab's. Figure 6 shows dense expression of all three proteins in the proliferating cell populations of germinal centers within secondary follicles of human tonsillar tissue. No significant expression of CDK2, KAP, or $\mathrm{HTm} 4$ was seen in the surrounding mantle zones of the follicles. All three proteins also demonstrated similar patterns of cellular colocalization with predominantly paranuclear/cytoplasmic staining, and to a lesser degree, nuclear staining, most evident with CDK2. This pattern of expression coincides exactly with cells that are in the active cell cycle, as evidenced by an identical staining pattern seen for Ki-67, a nuclear cell proliferation-associated antigen, expressed in all active stages of the cell cycle
(Figure 6d). Taken together, these findings suggest that these proteins are expressed specifically in actively cycling cells of the germinal centers within secondary follicles of human lymphoid tissues.

\section{Discussion}

We have shown that HTm4 is expressed in hematopoietic cells and tissues and highly regulated during the differentiation of hematopoietic stem cells. Our data also indicate that $\mathrm{HTm} 4$ expression is regulated during HSC progression through the cell cycle. This regulation seems to be both stage- and lineage-specific. Under certain cellular conditions, the expression of $\mathrm{HTm} 4$ is likely to be associated with the exit from cell cycle progression. As presented here, HTm 4 is detected in $\mathrm{G}_{0}$ quiescent stem cells and terminally differentiated hematopoietic cells of certain lineages. The biological role of $\mathrm{HTm} 4$ we elucidated is consistent with this observation.

Of particular interest to hematopoietic cell cycle control, we explored the interaction between HTm 4 and KAP and the effects on CDK2 phosphorylation and cell cycle progression. Our data indicate a physiologic HTm4-KAP interaction. HTm4 binds to KAP-CDK2 complexes through the binding of KAP to HTm 4 and can promote detectably the phosphatase activity of KAP. The C-terminal region of HTm 4 is required for these actions. The overexpression of $\mathrm{HTm} 4$ can cause cell cycle arrest at $\mathrm{G}_{0} / \mathrm{G}_{1}$ phase. We thus identify HTm 4 as a novel hematopoietic modulator for the $\mathrm{G}_{1}-\mathrm{S}$ cell cycle transition. We here put our findings in the context of previous pertinent research.
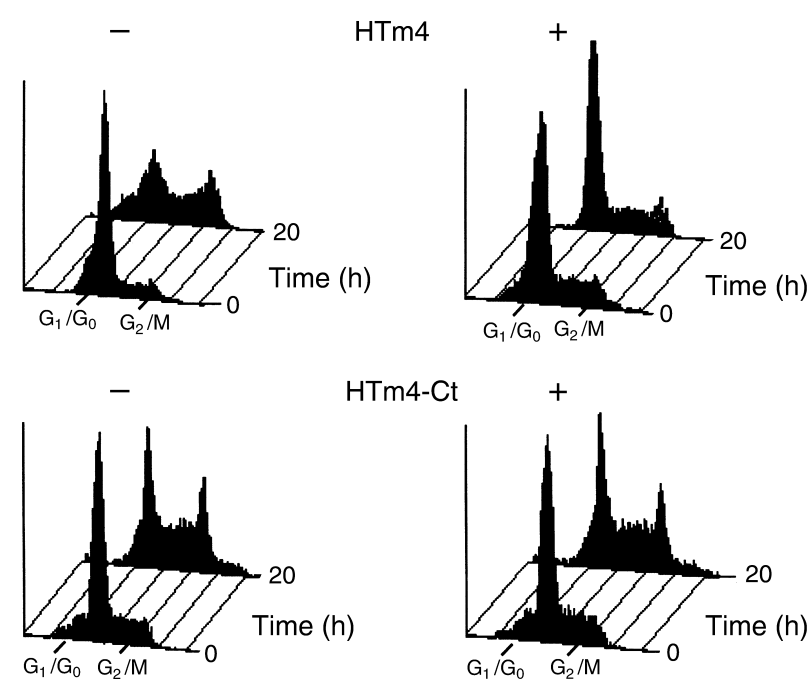

Figure 5

Cell cycle arrest is induced by the exogenous expression of $\mathrm{HTm} 4$. The upper panels are U937 with inducible Flag-HTm4 expression vector and the lower panels are with Flag-HTm4-Ct. +, induction of expression in the absence of Dox; -, no induction in the presence of Dox. Time intervals are given on the right. 0 hours, before the addition of FBS into the cultures; 20 hours, cells were cultured in the presence of serum for 20 hours after synchronization. $X$ axis shows cell cycle phase analyzed. Marked here are $\mathrm{G}_{0} / \mathrm{G}_{1}$ and $\mathrm{G}_{2} / \mathrm{M}$; in between (not marked) is $\mathrm{S}$ phase. Representative figures of at least five experiments. 

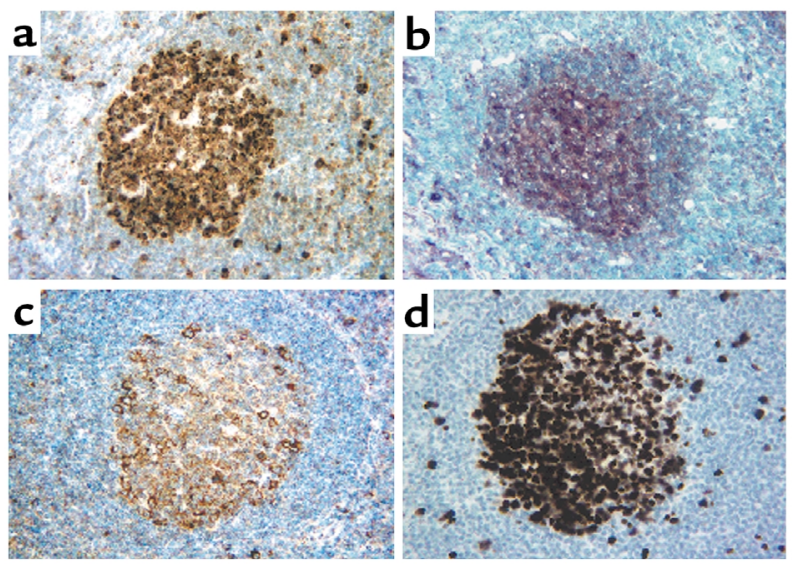

\section{Figure 6}

Immunoperoxidase staining of secondary follicles of the tonsil with CDK2, HTm4, KAP, and Ki-67. Sections of reactive tonsillar tissue are stained for expression of (a) CDK2, (b) HTm4, (c) KAP, and (d) Ki-67. Note the strong staining of the proliferating cells of the germinal center for all Ab's with absence of staining of the surrounding mantle zones. The cellular protein localization is predominantly nuclear for CDK2 and Ki-67 (a and d, respectively) with nuclear and paranuclear staining noted for $\mathrm{HTm} 4$ and $\mathrm{KAP}$ (b and $\mathbf{c}$, respectively). Magnification $\times 400$, HRP stain; hematoxylin was used as counterstain.

Our observations are consistent with the functional role of CDK2, which has been shown to be required for the transition from $\mathrm{G}_{1}$ to $\mathrm{S}$ phase of cell cycle (12). Previous research demonstrated that disruption of CDK2 activity by deactivation or overexpression may cause either $\mathrm{G}_{1}$ cell cycle arrest (35) or uncontrollable cell growth (36), respectively. Creating a situation similar to the deactivation of CDK2, the exogenous expression of $\mathrm{HTm} 4$ causes the progression of cell cycle to slow and therefore implicates a role for HTm 4 in cell cycle regulation. There are two possible modes of action, which might work in concert, for HTm4 to accomplish its functional role in vivo. First, the binding of HTm4 to KAP-CDK2 might enhance the phosphatase activity of KAP, which in turn would deactivate CDK2 at a faster rate. CDK2 is known to be such a potent kinase that it can inactivate the exogenously expressed retinoblastoma protein endogenously, and thus, prevent cell cycle arrest (37-39). The fine-tuning of CDK2 kinase activity may be essential for the balanced cell cycle progression. Second, because HTm4 is a transmembrane protein located in the perinuclear area, it might function to sequester KAP-CDK2 or KAPCDK2-cyclin E/A. This would keep these complexes out of their functional milieu and impact the transition from $\mathrm{G}_{1}$ to $S$ phase and $S$ phase progression of the cell cycle. Put in the context of previous findings, our data support the biological role of HTm4 as a regulator that modulates and possibly maintains a critical level of cyclin E/A-CDK2 kinase activity during the $\mathrm{G}_{1}-\mathrm{S}$ transition and $\mathrm{S}$ phase progression.

Our data on cell cycle arrest caused by the overexpression of exogenous HTm 4 are consistent with the find- ings of Yeh et al. (19). We have demonstrated that expression of HTm 4 stimulates the phosphatase activity of KAP and possibly sequesters CDK2 from its functional pathway, attenuating cell cycle progression at $\mathrm{G}_{0} / \mathrm{G}_{1}$. Yeh et al. found that the KAP produced by a hepatoma was defective in its ability to bind CDK2. This would result in a persistently activated CDK2 and uncontrollable cell growth. Interestingly, though, it has been shown that KAP is upregulated in both breast and prostate cancers (34). This apparent contradiction can be explained by the fact that in this study the integrity of KAP and its associated pathway were not confirmed. In addition, it should not be assumed that the same abnormality would yield an identical outcome in different cell contexts, because the status of other key cell cycle regulators may vary (3). When the downstream components are impaired, the resulting phenotypes cannot be overcome even when an upstream antagonist is overexpressed (40). The accompanying upregulation of regulators, along the same pathway as the damaged signaling component, might have been compensatory rather than causative (41).

Interestingly, our confirmation of the in vivo coexpression of KAP, CDK2, and HTm 4 in the proliferating cell populations of germinal centers within secondary follicles of human tonsils showed that these proteins are highly expressed in the actively cycling cells. As we have described both in the Discussion and Introduction, the observation that HTm 4 is expressed in both quiescent and actively cycling cells has basis and precedent. As described, p21 and p27 have been reported to have dual functions in cell cycle progression $(42,43)$. The same explanations for expression in proliferating and nonproliferating states that apply to p21 and p27 apply to HTm4: (a) expression of an inhibitory cell cycle regulator in response to proliferation or (b) an effect that is modified by the concentrations of other cell cycle regulators, both known and unknown $(3,42,43)$.

In conclusion, our research has provided substantial evidence for HTm 4 as a novel hematopoietic modulator for the $\mathrm{G}_{1}-\mathrm{S}$ cell-cycle transition. We have identified that HTm 4 binds to KAP and that HTm 4 forms a physiologic complex with KAP and CDK2. Exogenous expression of $\mathrm{HTm} 4$ promotes the dephosphorylation of $C D K 2$, leading to cell cycle arrest at $\mathrm{G}_{0} / \mathrm{G}_{1}$ phase. The immunohistochemistry data showing expression of HTm4, KAP, and CDK2 in the germinal centers of lymphoid tissues highlight the potential biological relevance of our observations in vivo.

\section{Acknowledgments}

This work was supported by NIH grant AI-43663 from the National Institute of Allergy and Infectious Diseases and by grant RSG-01-241-01-LIB from the American Cancer Society (to C.N. Adra); NIH grant AI-33100 (to M.H. Sayegh); RIKEN, Sagawa Foundation for Promotion of Cancer Research, Osaka Cancer Research Foundation, Welfide Medical Research Foundation (to T. Shirakawa); KO8 grant DK 02761 (to T. Cheng); 
FAPESP, Sao Paulo, Brazil (to J.L. Donato); NIHP30CA6516 (to J.L. Kutok); NIH-HL44851, NIHHL65909, and the Richard Saltonstall Charitable Foundation (to D.T. Scadden). This work was also supported by the Adra family and Dr. F. Jdeed, and is dedicated to the memory of Dr. Ramzi S. Cotran. We thank Professor T. Nakahata for his help and support. Assistance with preparation of manuscript was provided by Rachel Badovinac.

1. Metcalf, D. 1989. The molecular control of cell division, differentiation commitment and maturation in haemopoietic cells. Nature. 339:27-30.

2. Ogawa, M. 1993. Differentiation and proliferation of hematopoietic stem cells. Blood. 81:2844-2853.

3. Sherr, C.J. 1996. Cancer cell cycles. Science. 274:1672-1677.

4. Morgan, D.O. 1997. Cyclin-dependent kinases: engines, clocks, and microprocessors. Annu. Rev. Cell Dev. Biol. 13:261-291.

5. Johnson, D.G., et al. 1999. Cyclins and cell cycle checkpoints. Annu. Rev. Pharmacol. Toxicol. 39:295-312.

6. Israels, E.D., et al. 2001. The cell cycle. Stem Cells. 19:88-91.

7. Cheng, T., et al. 2000. Hematopoietic stem cell quiescence maintained by p21cip1/waf1. Science. 287:1804-1808.

8. Cheng, T., et al. 2000. Stem cell repopulation efficiency but not pool size is governed by p27(kip1). Nat. Med. 6:1235-1240.

9. Steinman, R.A., et al. 1998. Regulation of p21(WAF1) expression during normal myeloid differentiation. Blood. 91:4531-4542.

10. Taniguchi, T., et al. 1999. Expression of p21(Cip1/Waf1/Sdi1) and p27(Kip1) cyclin-dependent kinase inhibitors during human hematopoiesis. Blood. 93:4167-4178.

11. Sherr, C.J., et al. 1999. CDK inhibitors: positive and negative regulators of G1-phase progression. Genes Dev. 13:1501-1512.

12. Tsai, L.H., et al. 1993. The cdk2 kinase is required for the G1-to-S transition in mammalian cells. Oncogene. 8:1593-1602.

13. Brown, N.R., et al. 1999. Effects of phosphorylation of threonine 160 on cyclin-dependent kinase 2 structure and activity. J. Biol. Chem. 274:8746-8756.

14. Solomon, M.J. 1993. Activation of the various cyclin/cdc2 protein kinases. Curr. Opin. Cell Biol. 5:180-186.

15. Morgan, D.O. 1995. Principles of CDK regulation. Nature. 374:131-134

16. Poon, R.Y., et al. 1995 . Dephosphorylation of Cdk2 Thr160 by the cyclindependent kinase-interacting phosphatase KAP in the absence of cyclin. Science. 270:90-93.

17. Gyuris, J., et al. 1993. Cdi1, a human G1 and S phase protein phosphatase that associates with Cdk2. Cell. 75:791-803.

18. Hannon, G.J., et al. 1994. KAP: a dual specificity phosphatase that interacts with cyclin-dependent kinases. Proc. Natl. Acad. Sci. USA. 91:1731-1735.

19. Yeh, C.T., et al. 2000. Aberrant transcripts of the cyclin-dependent kinaseassociated protein phosphatase in hepatocellular carcinoma. Cancer Res. 60:4697-4700

20. Furukawa, Y., et al. 2000. Lineage-specific regulation of cell cycle control gene expression during haematopoietic cell differentiation. Br. J. Haematol. 110:663-673.

21. Adra, C.N., et al. 1994. Cloning of the cDNA for a hematopoietic cell-specific protein related to $\mathrm{CD} 20$ and the beta subunit of the high-affinity IgE receptor: evidence for a family of proteins with four membrane-spanning regions. Proc. Natl. Acad. Sci. USA. 91:10178-10152.

22. Adra, C.N., et al. 1999. Chromosome $11 \mathrm{q} 13$ and atopic asthma. Clin. Genet. 55:431-437.

23. Berardi, A.C., et al. 1995. Functional isolation and characterization of human hematopoietic stem cells. Science. 267:104-108.

24. Cheng, T., et al. 1996. Temporal mapping of gene expression levels during the differentiation of individual primary hematopoietic cells. Proc. Natl. Acad. Sci. USA. 93:13158-13163.

25. Taswell, C. 1981. Limiting dilution assays for the determination of immunocompetent cell frequencies. I. Data analysis. J. Immunol. 126:1614-1619.

26. Sambrook, J., Fritsch, E., and Maniatis, T. 1989. Molecular cloning: a laboratory manual. 2nd edition. Cold Spring Harbor Laboratory Press. Plainview, New York, USA.

27. Van Aelst, L., et al. 1996. Identification of a novel Rac1-interacting protein involved in membrane ruffling. EMBO. J. 15:3778-3786.

28. Crews, C.M., et al. 1992. The primary structure of MEK, a protein kinase that phosphorylates the ERK gene product. Science. 258:478-480.

29. Wang, Y., et al. 1991. byr2, a Schizosaccharomyces pombe gene encoding a protein kinase capable of partial suppression of the ras1 mutant phenotype. Mol. Cell Biol. 11:3554-3563.

30. Adra, C.N., et al. 1996. LAPTM5: a novel lysosomal-associated multispanning membrane protein preferentially expressed in hematopoietic cells. Genomics. 35:328-337.

31. Ling, Y.H., et al. 1996. Cell cycle-dependent cytotoxicity, G2/M phase arrest, and disruption of $\mathrm{p} 34 \mathrm{cdc} /$ cyclin B1 activity induced by doxorubicin in synchronized P388 cells. Mol. Pharmacol. 49:832-841.

32. Hass, R., et al. 1994. Characterization of human TUR leukemia cells: continued cell cycle progression in the presence of phorbol ester is associated with resistance to apoptosis. Eur. J. Cell Biol. 65:408-416.

33. Hass, R., et al. 1993. Resistance to phorbol ester-induced differentiation of a U-937 myeloid leukemia cell variant with a signaling defect upstream to Raf-1 kinase. Cell Growth Differ. 4:657-663.

34. Lee, S.W., et al. 2000. Overexpression of kinase-associated phosphatase (KAP) in breast and prostate cancer and inhibition of the transformed phenotype by antisense KAP expression. Mol. Cell Biol. 20:1723-1732.

35. Ghiani, C., et al. 2001. Inhibition of cyclin E-cyclin-dependent kinase 2 complex formation and activity is associated with cell cycle arrest and withdrawal in oligodendrocyte progenitor cells. J. Neurosci. 21:1274-1282.

36. Muller-Tidow, C., et al. 2001. Cyclin E is the only cyclin-dependent kinase 2 -associated cyclin that predicts metastasis and survival in early stage non-small cell lung cancer. Cancer Res. 61:647-53.

37. Lukas, J., et al. 1997. Cyclin E-induced S phase without activation of the pRb/E2F pathway. Genes Dev. 11:1479-1492.

38. Knudsen, E.S., et al. 1997. Dual mechanisms for the inhibition of E2F binding to RB by cyclin- dependent kinase-mediated RB phosphorylation. Mol. Cell Biol. 17:5771-5783.

39. Knudsen, E.S., et al. 1998. Inhibition of DNA synthesis by RB: effects on G1/S transition and S-phase progression. Genes Dev. 12:2278-2292.

40. Strobeck, M.W., et al. 2000. Restoration of retinoblastoma mediated signaling to Cdk2 results in cell cycle arrest. Oncogene. 19:1857-1867.

41. Weinstein, I.B. 2000. Disorders in cell circuitry during multistage carcinogenesis: the role of homeostasis. Carcinogenesis. 21:857-864.

42. LaBaer, J., et al. 1997. New functional activities for the p 21 family of CDK inhibitors. Genes Dev. 11:847-862.

43. Cheng, T., et al. 2001. TGF- $\beta 1$ mediates cell cycle arrest of primitive hematopoietic cells independent of $\mathrm{p} 21^{\mathrm{Cip} 1 / \mathrm{Waf} 1}$ or $\mathrm{p} 27^{\mathrm{Kip} 1}$. Blood. 98:3643-3649. 